\title{
MARGIT SLACHTA, UNE RELIGIEUSE DU TRAVAIL SOCIAL DANS LA VIE PUBLIQUE
}

\author{
MARGIT BALOGH
}

Académie des Sciences Hongroise, Institut de Sociologie

E-mail : balogh.margit@btk.mta.hu

\begin{abstract}
En 1958, le service des affaires intérieures du Ministère de l’intérieur hongrois a déclassé les archives liées à Margit Slachta. L’opposante politique, qui vivait encore aux Etats-Unis, n'était plus considérée comme dangereuse pour le socialisme en construction. En quelques dizaines d'années, elle était même peu à peu tombée dans l'oubli en laissant seulement l'image un peu falsifiée d'une " religieuse bigote ». Mais qui était véritablement Margit Slachta ? Il ne fait aucun doute qu'elle ne fut pas une personnalité banale. Si cela avait été le cas, elle n'aurait pas eu autant d'ennemis.
\end{abstract}

Mots-clefs : Margit Slachta, Edit Farkas, mission sociale, féminisme, nazisme/antinazisme, cardinal Mindszenty, émigration hongroise

\section{Son enfance et sa jeunesse}

Margit Slachta est née le 18 septembre 1884 à Kassa (Košice), dans une famille de la noblesse d'origine polonaise ${ }^{1}$. Ses aïeuls étaient de riches propriétaires terriens de la Haute Hongrie historique. Son père, Kálmán Slachta de Zadjel (zadjeli Slachta Kálmán), s’était marié en 1882 avec une demoiselle appartenant elle aussi à la classe des propriétaires terriens, Borbála Saárossy de Sáros. La vie du ménage fut prospère jusqu'à ce que le chef de famille, ayant dilapidé sa fortune, décidât d'émigrer en Amérique, en 1908, avec sa femme et trois de ses six enfants. Margit, qui était alors âgée de 24 ans, resta en Hongrie. Elle enseignait l'allemand et le français dans la ville de Győr et venait d'obtenir un poste de formateur à Budapest, dans l'institution de formation des maîtres de la rue Csalogány (Csalogány utca). 
Déjà, au cours de ses études, elle avait eu l'occasion d'entendre Edit Farkas s'exprimer sur les patronages et sur la protection des ouvrières, ce qui l'avait profondément impressionnée. En 1897, une veuve de la haute société, la comtesse Pál Pálffy (gróf Pálffy Pálné), avait fondé la première organisation sociale pour la protection des ouvrières dans un cadre chrétien, en s'appuyant sur des dames de sa condition, privilégiées, en un mot oisives. Il était alors à la mode de « s'abaisser » jusqu'aux classes inférieures, mais, pour le travail sérieux, l’enthousiasme des dames désœuvrées était insuffisant. On avait donc assez rapidement décidé de s'adjoindre l'aide de jeunes intellectuelles bien formées, parmi lesquelles se trouvait la jeune enseignante Edit Farkas qui accéda en 1906 à la vice-présidence de l'association. Tandis que la comtesse Pálffy croyait qu'il suffisait d'associer aux dames aristocratiques de jeunes femmes actives et cultivées, Edit Farkas quant à elle, réorienta le recrutement de simples collaboratrices temporaires vers celui de jeunes filles acceptant de consacrer leur vie et leur temps à cette activité. C'est ainsi que naquit l'idée d'une nouvelle organisation destinée à la cause féminine, la Mission du service social. Dès 1908, Margit Slachta rompit à son tour avec l'enseignement et fut la première candidate à la nouvelle mission de patronage lancée par Edit Farkas.

\section{La sœur de mission}

De la Mission sociale, Margit Slachta évolua bientôt vers la vie publique et la politique. Les membres de la mission se situaient au carrefour de la vie religieuse et de la vie dans le monde ; aujourd'hui, on dirait qu'elles faisaient partie d'un institut de vie communautaire et apostolique (en 1922, l'évêque leur confirma le statut de congrégation). Les sœurs ne faisaient pas de vœux publics, mais ce qu’on appelait alors des vœux privés. Pour Slachta, c'était le bon équilibre entre la proximité avec Dieu et l'immersion dans le siècle.

Vêtue d'un long pardessus et d'une large coiffe, comme c'était l'usage parmi les sœurs de la mission sociale, elle commença à donner ses premières conférences sur la condition de la femme et sur la nécessité d'union des femmes catholiques. En neuf ans, cette frêle et jolie jeune fille de 25 ans allait devenir la passionaria chrétienne de la cause féminine et du travail social. Moderne, dans le sens que l'on donnerait même aujourd'hui à ce terme, elle défendait ses idées avec une grande ardeur. Ottokár Prohászka, évêque de Székesfehérvár, la soutenait en raison même de ses méthodes inhabituelles : «j'aime cette jeune femme délicate et tendre et en même temps géniale, dont l'âme est d'une grande finesse ; elle est si radicale qu'elle peut sans difficulté adopter le mode de vie des classes les plus basses, elle est peu exigeante, capable par exemple de dormir par terre, s’il le faut, etc.... » Sans perdre haleine, elle enchaînait les conférences, récoltait 
des fonds, mettait en place des antennes de la Mission sociale en province. Elle ouvrit des écoles sociales, écrivit d'innombrables articles dans la presse sur la représentation des intérêts de la femme, sur le suffrage des femmes. Elle collabora au journal A Keresztény Nö ( « La femme chrétienne »), puis à la nouvelle version relancée en mars 1918, Magyar Nő (« La femme hongroise »), au sous-titre révélateur : A keresztény feminizmus lapja (« Journal du féminisme chrétien »). Le féminisme défendu par Slachta était à la fois favorable à la femme et centré sur la famille. Tout en mettant l'accent sur les obligations fondamentales de la femme en tant que génitrice, elle s'écartait des vues purement conservatrices assignant aux femmes le rôle de mère de famille à l'exclusion de tout autre fonction. Elle revendiquait des droits : le droit à l'éducation, le droit de vote, le droit à l'éligibilité. Elle était à la fois habile et volontaire, passionnée, inspirée et stimulante. C'est sans doute sa formidable aspiration au bien commun qui la motiva dans sa décision de jouer un rôle social d'importance. En 1918, elle s'inscrivit au Keresztényszociális Néppárt ("Parti populaire chrétien social »). Le 28 octobre de la même année, elle prenait la tête de la " section chrétienne féminine » du parti (Keresztény Női Tábor), avec l'intention de mettre en place tant l'organisation générale que les antennes locales d’un mouvement féminin chrétien, national, légitimiste et social.

\section{La première femme au parlement hongrois}

Les premières élections législatives en Hongrie, après la première guerre mondiale, furent tenues en 1920. Conformément aux attentes internationales, le suffrage fut général, égalitaire et secret - avec, toutefois, quelques restrictions concernant les femmes (par exemple, celle-ci étaient tenues, pour voter, de savoir lire et écrire dans une des langue du pays ${ }^{3}$ ). Slachta n'était membre du parti que depuis un an et demi, mais elle obtint à l'assemblée du Keresztény Nemzeti Egyesülés Pártja ( "Parti national chrétien unifié ») la majorité absolue pour être désignée comme candidate officielle à des élections partielles. Edit Farkas l'autorisa à participer aux élections, que Slachta gagna d'une confortable avance avec un total de 5471 bulletins exprimés en sa faveur, tandis que son opposant, l'ancien Ministre des affaires sociales, András Csilléry, obtenait 3642 votes et que les deux autres candidats en présence ne dépassaient pas le millier ${ }^{4}$. Margit Slachta devenait ainsi la première femme élue au parlement de Hongrie, elle était en quelque sorte la première "mère de la nation ". Du reste, son apparition sur la scène politique fut accueillie avec des sentiments mitigés. Certains célébrèrent la victoire de l'émancipation, d'autres s'agitèrent au nom du principe que l'arène politique n'était pas un lieu convenable pour les femmes. 
D’ailleurs, ceux-là se trompaient qui croyaient que la voix de la jeune femme serait nécessairement embarrassée, aurait du mal à porter au sein de l'assemblée. Lors de son premier mandat, qui commença le 25 mars 1920 et se termina le 16 février 1922, elle prit la parole 28 fois, s’exprimant sur non moins de 67 problèmes majeurs. Elle donna son premier discours le 23 avril 1920. Le correspondant du journal social démocrate Népszava racontait que « tout en avançant les vérités qu'elle avait apprises des femmes socialistes, elle fut constamment interrompue par les politiciens mâles qui plaisantaient, imbus de leur supériorité5. » Elle aborda tour à tour la question et la situation féminine, la situation sociale, le suffrage universel, proposant par exemple qu'aux femmes (et aux jeunes hommes âgés de moins de vingt ans) fût interdit l'accès aux détaillants d'eaux de vie, car la vue des hommes pris d'ivresse n'était pas favorable à l'édification de l'âme féminine ${ }^{6}$. Elle proposa la modification des lois relatives au travail féminin, l'embauche de « contrôleur » de sexe féminin dans l'industrie, la garantie institutionnelle de la protection de la maternité, l’octroi de deux semaines de congés payés aux ouvriers de l'industrie, la limitation de la mise en nourrice, alors en plein développement au détriment de l'allaitement maternel, enfin, la révision du droit pénal des mœurs. Les membres du parlement se rendirent compte assez vite qu'il ne s'agissait pas de ricaner de leur consœur avec sarcasme ou supériorité. Ses discours, ses propositions, ses positions démontraient qu'elle était une femme cultivée et profondément sensible à la chose sociale.

Et pourtant, l'historiographie la traite avec une grande injustice. Non sans quelque approximation soigneusement entretenue, le nom de Slachta est en effet associé avec la loi XXVI de 1920 sur la défense de la propriété, de la morale et des personnes, demeurée célèbre sous le nom de « loi du bâton ». Or elle n’était pas présente lors des discussions et du vote de cette loi. Elle était en vacances. L'origine de la confusion tient au fait qu'une autre loi en discussion à la même époque prévoyait elle aussi la peine du bâton, la loi XV de 1920 dont l'objet était de renforcer la punition du profit illicite. Lors des discussions sur la loi XV, Slachta s'était rangée derrière les partisans du bâton et avait même proposé son extension aux femmes. Entre autres, elle affirmait être elle-même arrivée à « cette étrange constatation que c'étaient les femmes et non les hommes qui étaient responsables du profit illicite ». Selon elle, la conquête de l'égalité pour les femmes devait passer par les avantages, mais aussi par les inconvénients. D’autre part, la punition du profit illicite par la peine du bâton n'était pas spécialement critiquée, y compris dans la presse de gauche, où l'on commença seulement à la stigmatiser en première page quand il apparut qu'elle pourrait être appliquée contre les associations de gauche. (Le parlement commençait justement à discuter, pour la protection des intérêts supérieurs de l'État et de la société, d'une proposition de loi prévoyant l'interdiction du parti communiste - concrétisée l'année suivante par la loi III de 1921). Avec le temps, des articles tendancieux firent peu à peu 
l'amalgame entre les restrictions imposées à la gauche, la peine du bâton et Margit Slachta. Plus tard, l'intéressée allait d'ailleurs elle-même réviser son jugement sur la question. Mais l'historiographie allait tout de même conserver cette étrange obsession pour son prétendu goût de la peine du bâton.

Le mandat parlementaire de Slachta prit fin le 16 février 1922. Edit Farkas ne l'autorisa pas à se présenter aux nouvelles élections. Les deux femmes s'étaient éloignées l'une de l'autre, car leurs conceptions respectives de l'avenir du pays étaient profondément différentes, quoique toutes deux monarchistes : Edit Farkas était partisan de la libre élection du roi et entretenait des relations amicales avec la famille du régent Horthy. Slachta, quant à elle, était légitimiste et le demeura jusqu'à sa mort, c'est-à-dire qu'elle souhaitait que le trône fût réservé à un membre de la Maison Habsbourg, Charles IV, puis, après la mort de ce dernier, à son fils Othon, selon les règles strictes de dévolution inscrites dans la Pragmatica sanctio de $1723^{7}$.

D’autre part, la conférence des évêques hongrois avait rejeté plusieurs fois les constitutions de la Mission sociale, exigeant surtout la cessation de toute activité politique par leurs membres. Edit Farkas modifia l'inspiration bénédictine initiale de sa règle pour y infuser un certain esprit jésuite, non sans conserver une part de méticulosité médiévale dans l'organisation quotidienne de la communauté, formant un mélange inédit. Du reste, les obligations de la vie communautaire (la prière, le jeûne, la discipline dans la maisonnée, etc...) n'étaient pas faites pour Slachta, qui refusait d'être une religieuse aux mains liées par une multitude de règles. Elle souhaitait simplement faire le vœux de s'immerger dans le monde, qu'elle considérait comme le lieu le plus adapté pour un travail social efficace et institutionnalisé.

Entre les deux femmes, le conflit larvé sur la nature de l'engagement politique s’aggrava jusqu'à ce que Margit Slachta finît par quitter la Mission, le 5 mai 1923, accompagnée de quelques autres religieuses. Les parcours de ces deux femmes de grande valeur en dépit de leurs grandes divergences, n’allaient plus jamais se rejoindre. Quelques jours plus tard, le 12 mai, Slachta fonda la Société des sœurs du service social, qu'elle allait diriger pendant quarante ans, jusqu'en 1963. Avec ses compagnes, elle abandonna le voile bleu et adopta un voile gris, c'est pourquoi on les a appelées les « sœurs grises ». Après avoir franchi les premières difficultés liées à l'établissement de la Société, Slachta reprit ses activités politiques, en 1927.

Revenons donc au parti politique, la Section chrétienne féminine. Depuis sa fondation en 1918, on avait l'habitude d'organiser au cours de l'assemblée annuelle du parti une " parade » qui consistait en séances de formation et discussions informelles. Slachta donnait généralement un discours sur les activités réalisées au cours de l'année, ainsi que sur les orientations de fond du parti. Après l'éclatement de la deuxième guerre mondiale, elle entreprit de renforcer l'esprit 
chrétien de résistance morale : lors de la Parade de 1939, elle évoqua notamment la question juive en affirmant que « toute injustice agit comme un boomerang et revient vers celui qui la commet ${ }^{8}$. " À cette occasion, le parti adopta notamment une motion qui fut présentée au Président du conseil : à savoir que les prêtres ou religieux des ordres féminins et masculins d'origine juive soient exemptés de l'application des lois juives (antisémites). Le 6 janvier 1942, elle s'exprima publiquement contre le nazisme. L'ambassadeur d'Allemagne protesta auprès du Ministère hongrois des affaires étrangères, tandis que la presse libérale et radicale hongroise donnait au contraire un écho favorable ${ }^{9}$. À partir de 1942, le parti organisa à travers le pays des « séminaires de vision du monde » afin d'apporter le contrepoids des valeurs chrétiennes à la propagande hitlérienne ${ }^{10}$.

\section{Margit Slachta, la juste}

Quand l'antisémitisme se répandit dans l'opinion hongroise, Slachta le repoussa, pour sa part, de la plus ferme manière. À mesure que s'approchait l'hitlérisme, ses positions théoriques prirent une forme plus active. Elle écrivit et diffusa d'innombrables tracts et pétitions, intervint auprès des pouvoirs publics. Elle publia plusieurs séries d'articles, en particulier dans le journal A Lélek Szava (« La parole de l'âme »), qui fut l'un des premiers organes de presse supprimés après l'invasion allemande ${ }^{11}$. Accompagnée de onze religieuses de la Société, elle présenta devant le Prince primat, Mgr Jusztinián Serédi, une protestation contre les lois juives : «l'enseignement de notre Eglise nous interdit de nous associer avec les conceptions sur lesquelles se fonde la législation hongroise pour persécuter les juifs, moins encore avec celles qui, ignorant la sainteté du sacrement du baptême, considèrent à certains égard des chrétiens comme des juifs, et bien moins encore nous pouvons accepter que ces lois soient assez imparfaites pour qualifier de juifs des personnes qui ont dédié leur vie entière au service des âmes au sein de l'Eglise ${ }^{12}$.»

En 1939, les catholiques convertis avaient fondé une institution de défense, l'Association hongroise de la Sainte Croix, dont Margit Slachta était vice-présidente. Au printemps 1942, les premières nouvelles de la déportation des juifs de Slovaquie parvinrent à l'association. " Je suis rentrée à la maison avec l'âme dévastée - écrivait-elle - ce qui me tourmente, c'est que j'ai le sentiment d'être moi aussi coupable, si je ne fais pas tout pour empêcher ce désastre ${ }^{13}$. "

Les protestations de Slachta ne furent entendues ni par l'Etat hongrois, ni par l'Église. Après l'annonce, le 8 février 1943, que la Slovaquie allait bientôt commencer la liquidation de tous les juifs, Slachta cessa toute tergiversation. Elle se rendit à Rome où elle parvint à obtenir une audience papale le 11 mars 1943, obtenant que Pie XII somme les sept évêques slovaques de protester auprès du prési- 
dent de la République et du gouvernement de Slovaquie et fassent en sorte qu'une lettre pastorale de protestation fût lue dans toutes les églises de Slovaquie ${ }^{14}$.

Sa foi chrétienne et son humanisme stimulaient son engagement dans une activité qui lui faisait risquer sa vie. Les sœurs de la Société restaient volontairement à ses côtés. L'enseignement spirituel des religieuses contenait la disposition au don de soi-même et le vœu d’obéissance, mais sans préjudice du l'examen de conscience. C'est-à-dire que chacune pouvait choisir librement : devait-elle risquer sa propre vie pour sauver celle des autres ? Outre les sœurs de la Société, Slachta œuvrait avec tous ceux qui étaient prêts à s’engager. Par exemple, la militante sociale démocrate Sára Karig imprimait des certificats de baptême et falsifiait des tampons qu'elle remettait à Slachta ${ }^{15}$. Les sœurs grises sauvèrent ainsi un millier de juifs. Certaines d'entre elles y perdirent leur propre vie : Sára Salkaházi fut exécutée et jetée dans le Danube le 27 décembre 1944 par les Croix fléchées, avec une catéchiste, Vilma Bernovits, et quatre enfants réfugiés.

\section{Après la deuxième guerre mondiale}

Début 1945, Slachta fut accueillie, non sans débat houleux, par les dirigeants du Parti démocrate chrétien populaire. Mais son nom était associé au parti chrétien d'avant-guerre qui avait perdu tout crédit. De plus, sa propre position politique était jugée éloignée de celle du Parti populaire. Les différences d’opinion finirent par provoquer la scission : le comte József Pálffy, président du parti, expulsa non seulement les « dissidents » modernistes (qui allaient former le Parti démocrate populaire), mais aussi l'aile conservatrice, plus proche du clergé, qui tint une assemblée générale en se donnant Slachta pour vice-présidente. La Commission nationale générale (Országos Nemzeti Bizottság) autorisa le Parti démocrate populaire, qui affichait des positions progressiste jugées en phase avec les exigences de la politique internationale du moment, mais il n'autorisa pas l'aile conservatrice à faire campagne en tant que parti aux élections législatives de 1945.

Quant à Slachta, elle se présenta comme candidate individuelle et entra finalement au parlement sur la liste du Parti démocrate bourgeois (Polgári Demokrata Párt). Son engagement était considéré avec suspicion par de nombreux catholiques : puisque la persécution des juifs n’était plus d’actualité, pourquoi devait-elle s’allier avec le parti bourgeois qui continuait à représenter ? D’autre part, beaucoup de ses confrères au parlement - pas seulement les marxistes, mais aussi nombre de croyants chrétiens - la considéraient comme représentante d'un cléricalisme dépassé. Ses discours furent accueillis avec des exclamations sarcastiques, parfois vulgaires. La vieille question de la peine du bâton suscita des accusations récurrentes. On s’efforçait ainsi d’anéantir, du moins de contester le grand capital moral que Slachta avait acquis en sauvant des vies pendant la guerre. 
Elle défendait des valeurs conservatrices. Au parlement, elle s’opposa à la proposition de loi sur le régime républicain, favorisant - d'accord avec le cardinal primat József Mindszenty - l'organisation d'un référendum sur la question qu'elle proposait de soumettre après la conclusion de la paix (on espérait encore que le pays recouvrirait alors sa souveraineté). Slachta était attachée aux anciennes structures et aux autorités héritées du passé. Le royaume promettait la même stabilité, les mêmes structures solides que l'Eglise ; de plus, il entretenait des liens étroits avec le catholicisme. Mais la réalité était incontournable : l’Union soviétique avait étendu son influence sur la moitié de l'Europe. Au terme de cette deuxième guerre mondiale, il y avait quelque chose de véritablement incongru dans l'idée d'un royaume en Europe centrale. Mais Slachta refusait de croire qu'un changement de régime pût mettre la Hongrie dans une meilleure position lors des négociations de paix.

En 1947, elle participa de nouveau aux élections générales et fut élue dans le cadre de la Section chrétienne féminine qu'elle venait de réactiver. Pendant la campagne, elle mit l'accent sur l'organisation des femmes sur une base chrétienne, nationale et sociale, sur l'amélioration de la situation économique et politique des femmes et sur la diffusion de l'intelligence féminine dans la vie publique : toutes les idées qu'elle n'avait cessé de défendre. La protection des enfants, des femmes, de la famille étaient des thèmes sympathiques, mais, après un événement comme la deuxième guerre mondiale, c'était insuffisant. La coalition au pouvoir, menée par le Parti des petits propriétaires (Kisgazda párt), avait déjà passé un grand nombre de lois sociales, il était impossible de gagner les votes des femmes en s'appuyant seulement sur la nature spécifique des femmes et la vision du monde chrétienne. La campagne du parti de Slachta éveilla peu d'écho, ni dans la coalition, ni dans les partis de l'opposition. (Aujourd'hui encore, il serait peu judicieux de fonder en Hongrie un parti politique sur des questions de genre.) En 1947, la Section féminine chrétienne exerça peu d'influence, mais, avec 1,4\% des suffrages, elle parvint tout de même à envoyer quatre représentants au parlement.

Les interventions de Slachta, en 1947, furent principalement consacrées à la question de l'État de Saint Étienne, en tant que symbiose intacte du christianisme et de la monarchie, et au maintien de la " constitution historique » de la Hongrie. Nous connaissons les nombreux efforts du gouvernement en vue de réduire progressivement l'indépendance de l'Église catholique. Le 5 février 1947, lors du débat parlementaire sur l'introduction du caractère facultatif de l'enseignement religieux à l'école, conformément à la mise en application de la liberté de conscience, Slachta prononça un long discours en faveur de son caractère obligatoire. (Un député indépendant, qui venait de quitter le jour même le Parti des petits propriétaires, György Parragi, allait dire à ce propos : "s'il y a un homme dans cette assemblée, c’est bien elle ! »). 
Le glissement de la vie politique hongroise vers la gauche finit par rendre impossible la poursuite de son activité politique dans le pays. Son discours du 28 octobre 1947 fut considéré comme préjudiciable à la politique extérieure du pays (elle y avait condamné l’Union soviétique et la Yougoslavie). Le 30 octobre, sur proposition du comité de l'immunité parlementaire, elle fut exclue de l'assemblée pour une durée de soixante jours. Lors de son dernier discours - constamment interrompu - en date du 16 juin 1948, elle s’opposa avec véhémence à la proposition de loi sur la nationalisation des écoles religieuses. Après le vote de la loi, les représentants des partis gouvernementaux entonnèrent l'hymne national, mais Margit Slachta resta assise. Le comité de l’immunité décida de prononcer une nouvelle fois son exclusion, mais pour une durée de deux fois six mois ${ }^{16}$.

Connue depuis toujours pour sa droiture, Margit Slachta finit par être rejetée pour son intransigeance. Slachta ne pouvait défendre le christianisme que dans son intégralité, elle était inapte à formuler le moindre compromis sur les principes. Ses luttes imprégnées d'équité, marquées par sa vision du monde chrétienne étaient irrémédiablement condamnées à l'échec dans cette époque féroce. Etait-elle, elle-même, après 1945 la même personne qu'elle avait été en 1920 ? Encore jeune femme au temps de sa première expérience parlementaire, elle était déjà une dame d'âge mûr lors de la seconde. Mais il n’y a pas deux Margit Slachta, seulement deux périodes historiques profondément différentes. Si elle avait pu représenter en 1920 une pensée catholique moderne, trois décennies plus tard, elle n'était déjà plus en mesure de remarquer les changements survenus même au sein du camp catholique. Elle restait fidèle à ses anciennes opinions.

L'été 1948 venait d'être marquée par l'exclusion de Slachta des travaux parlementaires. La deuxième partie de l'année le fut par la lutte contre le « mindszentysme » qui se termina, comme on le sait, par l'enfermement du prince primat le 26 décembre, à l'encontre de toute autre solution plus diplomatique. De son lieu de détention, le cardinal fut autorisé à envoyer deux courtes lettres à Margit Slachta, dans lesquelles il demandait quelques menus services. Slachta considéra ces lettres avec suspicion, croyant - sans doute à juste titre - qu'il s'agissait d'une provocation. Craignant une prochaine arrestation, elle se cacha dans un couvent dominicain à partir de janvier 1949. À l'origine, elle avait prévu de faire campagne pour les élections de 1949, en réalité, c'est à peine si elle vota elle-même le 15 mai. Finalement, le 23 juin 1949, à l'aube, elle traversa la frontière en direction de l'Autriche avec sa sœur cadette, d'où elle émigra aux ÉtatsUnis le 16 septembre sous le pseudonyme d’Etelka Tóth. 


\section{Dans l'émigration}

De son exil, elle continua à correspondre avec des connaissances en Hongrie sous le pseudonyme de Margit Nemes ; sous celui de Borbála Nemes, elle collabora à Radio Free Europe. En 1951, elle revint à Vienne avec l'espoir, qui s'avéra vain, de revoir son pays natal, mais elle reprit finalement le chemin des Etats-Unis le 5 mai 1953, cette fois-ci définitivement et sous son vrai nom.

Au cours des premières années d'émigration, elle s'efforça de rester active. À l'une de ses sœurs, elle écrivait en 1954 : « Tu ne peux imaginer combien je suis débordée. Sans cesse, je dois m’occuper de ma survie et de celle de mes sœurs, sans cesse résoudre des problèmes, écrire des lettres... Je suis vidée, ma tête refuse de se souvenir. Rappelle-toi tout de même que j'ai 70 ans. Je ne me suis pas assise sur un divan pour me couper les ongles depuis dix ans ${ }^{17} \ldots$.. En 1956, elle était déjà affaiblie, mais elle fit de son mieux pour venir en aide aux réfugiés.

Avec le temps, elle s'était retirée de la politique, mais elle continuait à entretenir une correspondance avec quelques " anciens combattants ». Béla Varga, ancien président du parlement, lui écrivit par exemple en 1959 : « Je sais que dans ce monde étranger où je suis loin de ma patrie, il y a près de moi la meilleure amie de toute ma vie ${ }^{18}$. » Deux ans plus tard, il la mettait encore en garde contre toute idée de retour en Hongrie : " Pour tout l'or du monde, n'essayez pas ${ }^{19} .$. »

Même au sein de sa propre sororité, elle eut à subir des conflits douloureux qui reflètent étrangement son sort personnel en 1923. Les sœurs de Los Angeles et du Canada finirent par rompre avec elle. Ses dernières années furent celles du dépouillement total. Son corps fatigué finit par venir à bout de ses capacités. Elle passa le 6 janvier 1974 à l'âge de 90 ans, à Buffalo, dans une maison de la Société des sœurs du service social.

Le personnage de Margit Slachta représente des valeurs et une attitude exemplaires, indépendante de toute position politique, dominant toutes les époques, tous les pouvoirs et toutes les visions du monde. Elle fut une personne extrêmement complexe et compliquée. Et pourtant simple, par ailleurs, car elle s'est constamment soumise au commandement d'amour du Christ. Elle se sentait à la fois revêtue d'une responsabilité envers les autres et envers la nation.

Onze ans après sa mort, en 1985, elle reçut à titre posthume la distinction des Justes de l'Etat d'Israël et l'on planta un arbre à sa mémoire dans le parc de Yad Vashem. Le 15 mars 1995, Margit Slachta reçut du gouvernement hongrois la médaille du souvenir (emlékérem), conjointement avec la Société des sœurs du service social, puis, le 7 mai 1995, la médaille du courage de la République de Hongrie (Magyar Köztársaság Bátorság érdemjel). Mais ce n’est pas pour obtenir des décorations qu'elle a tant lutté et souffert : la Société des sœurs du service social n'est pas morte, elle accueille encore de jeunes sœurs, la relève est arrivée pour le XXI siècle. 


\section{Notes}

1 Palkó Magda, «Slachta Margit », Politikuspályák [Parcours politiques], Budapest, 1984, p. 146.

2 Prohászka Ottokár, Naplójegyzetek 3. [Journal], Szeged-Székesfehérvár, 1997, p. 130. Note du 11 janvier 1920.

3 Magyarországi Rendeletek Tára [Répertoire des décrets hongrois], 1919, p. 879.

$4 \quad$ Budapesti Hírlap, 28 mars 1920, p. 5.

5 Népszava, 24 avril 1920, p. 1.

6 Magyar Nö, année IV. (début IX.), n², 26 mars 1921.

7 NdT : Le parti de la libre élection considérait la déclaration de Charles IV à Eckartsau, le 13 novembre 1918, comme une abdication, il fallait donc, selon lui procéder à l'élection d'un nouveau roi, conformément aux anciennes traditions hongroises et surtout aux luttes pour l'indépendance nationale ayant culminé en 1848-49.

$8 \quad$ Pesti Hírlap, 8 janvier 1939, p. 19.

9 Pesti Hírlap, 8 janvier 1942, p. 7 ; Magyar Nemzet, 8 janvier 1942, p. 8 ; Nemzeti Újság, 3 janvier 1942, p. 3.

10 Sorakozzál a Front védelmére [Engagez-vous avec le Front], [Budapest] 1943, 4 pages.

11 NdT : l'invasion de la Hongrie par l'armée allemande eut lieu le 19 mars 1944.

12 Archives primatiales (Esztergom) 4843/1941. 8 juillet 1941.

$\underline{13}$ Lettre à l'archevêque de Kalocsa, Mgr Gyula Zichy, 27 avril 1942. Archives de la Société des sœurs du travail social (Szociális Testvérek Társasága Történeti Irattára). Plus loin : « SzTT$\underline{\mathrm{TI}} \Perp$

14 Mona Ilona, Slachta Margit, Budapest, 1997, p. 153.

15 Interview de Sára Karig dans le documentaire intitulé Credo (réalisation : Ágnes Tölgyesi), 20 mai 1995.

16 SzTTTI, lettre de Margit Slachta à Alice Slachta (résidant alors à Shanghai), Budapest, sans date.

17 SzTTTI, Lettre de Margit Slachta à Irén Slachta. Kneipp Spring, 29 mars 1954.

18 SzTTTI, Lettre de Béla Varga à Margit Slachta. 11 décembre 1959.

19 SzTTTI, Lettre de Béla Varga à Margit Slachta. 9 mars 1961. 